\title{
Upregulation of the Kank1 gene-induced brain glioma apoptosis and blockade of the cell cycle in G0/G1 phase
}

\author{
XIAOHANG GUO ${ }^{1}$, WENHAI FAN ${ }^{2}$, XINCHAO BIAN $^{3}$ and DIHUI MA ${ }^{1}$ \\ ${ }^{1}$ Department of Neurology, The First Hospital of Jilin University, Changchun 130021; \\ ${ }^{2}$ Department of Neurosurgery, The Affiliated Zhongshan Hospital of Dalian University, Dalian 116001; \\ ${ }^{3}$ Department of Neurosurgery, Zibo Central Hospital, Zibo 255000, P.R. China
}

Received November 12, 2013; Accepted December 19, 2013

DOI: $10.3892 /$ ijo.2014.2247

\begin{abstract}
The Kank1 gene is one of the important members of the Kank gene family. As an important adaptor protein, Kank1 plays a significant role in the genesis and development of many malignant tumors. It was recently discovered that the Kank1 gene is a new cancer suppressor, and its expression is significantly downregulated or it is not expressed in kidney cancer, bladder cancer, prostate cancer, lung cancer and breast cancer. However, no report on the role of Kank1 in the genesis of brain glioma is available to date. In this study, we found significantly lower expression of the Kank1 gene in human brain glioma cells compared to the other cells evaluated. We used RNA interference techniques to silence Kank1 gene expression and found acceleration of tumor cell proliferation. However, when the Kank1 gene was upregulated, cell apoptosis occurred and the cell cycle was blocked in the G0/G1 phase. Also, we found that upregulating the Kank1 gene may result in the change of mitochondrial membrane potential, and the regulation of Bax and Bcl-2 may promote the mitochondria to release cytochrome $\mathrm{C}$ so as to activate Caspase- 9 and -3 . Thus, the human brain glioma apoptosis induced by upregulation of the Kank1 gene is closely relevant to the mitochondrial pathway.
\end{abstract}

\section{Introduction}

Brain glioma is the most common malignant tumor of the primary tumors in the central nervous system and the main cause of death of the patients with intracranial tumor $(1,2)$. So far, the clinical approaches to treat brain glioma include surgery, radiotherapy, and chemotherapy (3-6), but the overall curative effect is far from satisfactory. Moreover, our knowledge on the molecular mechanism of the brain glioma is rather limited and active exploration is needed. To clarify the role a certain gene plays in the genesis of brain glioma, is of

Correspondence to: Dr Dihui Ma, Department of Neurology, The First Hospital of Jilin University, 71 Xinmin Avenue, Changchun 130021, Jilin Province, P.R. China

E-mail: dihuima@163.com

Key words: Kank1 gene, glioma, increase, apoptosis, mitochondria significant importance to understand the malignant biological behavior of brain glioma, and may provide a reliable molecular target for the molecular targeting treatment in the future.

As an important adaptor protein, and mainly distributed in the cytoplasm, Kank1 often forms a compound with $\beta$-catenin to shuttle among the nucleoplasm to regulate the distribution of $\beta$-catenin, playing a key role in the genesis and development of many malignant tumors (7). The expression of Kank1 is very extensive and is found in many normal tissues (such as the epithelial cells of the kidney tubules, the glance cells of the colon, the stomach and other digestive organs). However, its expression is downregulated or missing in the tissues of kidney tumors, lung tumors, as well as the corresponding tumor cell lines (8-10). It has been discovered previously that one of the factors to cause abnormal gene expression and loss of function is the great loss or mutation of tumor suppression genes, where the deficiency of chromosome $9 \mathrm{p}$ has been reported in many kinds of tumor and other diseases. This means the deficiency of Kank1 gene locus or the abnormal gene expression due to the change in epigenetics may be relevant to the genesis and development of the diseases, especially closely relevant to the genesis of many human tumors. Those carcinogenesis mechanisms have been proven in kidney cancer, cervical cancer, bladder cancer, prostate cancer, lung cancer and breast cancer (8-12). Our research revealed that upregulating Kank1 gene may cause the change of Bax and Bcl-2 as well as the blockade of the cell cycle in phase G0/G1. The translocation of Bax and $\mathrm{Bcl}-2$ may result in the change of mitochondria membrane potential to release cytochrome $\mathrm{C}$ so as to activate the Caspase family for final cell apoptosis $(13,14)$.

The objective of this study is to investigate the impact of Kank1 gene on the cell proliferation, apoptosis and the cell cycle, and to explore the signal transduction pathway of cell apoptosis induced by upregulating the Kank1 gene.

\section{Materials and methods}

Antibodies and reagents. Kank1, Bax, Bcl-2, Caspase-3, Caspase-9, CDK4, CDK6 and Cyclin D1 antibodies were from Cell Signaling Technology, Inc. (Danvers, MA, USA). DMEM culture medium was from Sigma Co. (St. Louis, MO, USA). Fetal calf serum was from Gibco (Grand Island, NY, USA), siRNA and plasmid were synthesized by GenePharma Co., 
Ltd. (Shanghai, China), RT-PCR kit [Takara RNA PCR kit (AMV) Ver.3.0] was from Takara Biotechnology Co., Ltd. PI was from Sigma. The Annexin V-fluorescein isothiocyanate (FITC) kit was from Bio-Rad (Hercules, CA, USA).

Cell culture. Human brain glioma U87 and U251 cells (ATCC, Manassas, VA, USA) were cultured in the DMEM culture medium containing $10 \%$ fetal calf serum, $100 \mathrm{U} / \mathrm{ml}$ penicillin and $100 \mu \mathrm{g} / \mathrm{ml}$ streptomycin, and then cultured in the incubator containing $5 \% \mathrm{CO}_{2}$ at $37^{\circ} \mathrm{C}$.

Cell viability. Human brain glioma cells were inoculated in a 96-well culture plate with the density of $1 \times 10^{5} / \mathrm{ml}$ and used in treatments when the cells grew to log phase. Six double wells were set for each experimental group. Each well was added with $20 \mu \mathrm{l}$ of MTT $(5 \mathrm{mg} / \mathrm{ml})$ and cultured in $\mathrm{CO}_{2}$ incubator for $4 \mathrm{~h}$ before the culture solution was discarded. DMSO $(150 \mu \mathrm{l})$ was added to each well at room temperature with oscillation for $10 \mathrm{~min}$ and a microplate reader was used for analysis.

Cell transfection. Human brain glioma cells were inoculated in a 6-well culture plate with the density of $5 \times 10^{5} / \mathrm{ml}$. Transfection was carried out when the cells grow to higher than $70 \%$ confluence. Negative oligonucleotides was used as the control group. The culture medium was replaced to medium without serum or antibiotics, siRNA and Lipofectamine 2000 was added, respectively, adjust the preset concentration following the instructions of the transfection reagent. The culture plate was placed in $\mathrm{CO}_{2}$ incubator for 4-6 $\mathrm{h}$ after siRNA transfection, and the culture continued using medium with serum. Following plasmid transfection the transfected cell line was screened. Resistant monoclone was established 3 weeks after the screening, the selected monoclone was expand in culture to establish the overexpressing Kank1 brain glioma cell line. RT-PCR and western blot analysis were performed.

RNA extraction and RT-PCR analysis. Total RNA was extracted from the brain glioma cells referring to the instructions from RNAiso ${ }^{\text {TM }}$ Plus (Takara, Japan). After determining total RNA concentration, RT-PCR reagent (Takara) was used for RT-PCR. Kank1 and $\beta$-actin gene primer were designed and synthesized by Invitrogen. Kank1 gene forward primer, 5'-GCACCCTGTCGTCTATCAACTC-3'; reverse primer, 5'-CTGCTGATTGGCTTTCCTTCT-3'. $\beta$-actin gene forward primer, 5'-CTGGGACGACATGGAGAAAA-3'; reverse primer, 5'-AAGGAAGGCTGGAAGAGTGC-3'. PCR reaction, $50 \mu \mathrm{l}$ at the following reaction conditions: $94^{\circ} \mathrm{C}$ for $2 \mathrm{~min}$, $94^{\circ} \mathrm{C}$ degeneration for $30 \mathrm{sec}$, annealing at $60^{\circ} \mathrm{C}$ for $30 \mathrm{sec}$, extension at $72^{\circ} \mathrm{C}$ for $30 \mathrm{sec}$, for a total of 31 cycles. The PCR product was electrophoresis with $1.5 \%$ agarose gel, then scanned and analyzed with gel imaging system (G: BOX Chemi XR5; Syngene, Cambridge, UK).

Analysis of apoptosis with flow cytometry. Trypsin was used to digest and collect the treated brain glioma cells, and the cells were suspended, with PBS to prepare a single cell suspension. Annexin V and PI staining fluid were added, respectively, in accordance with the Annexin V-fluorescein isothiocyanate (FITC) kit (Bio-Rad). Staining followed for $15 \mathrm{~min}$ at room temperature before the analysis with flow cytometry (BD Biosciences, Franklin Lakes, NJ, USA) for apoptosis.

Hoechst 33342 staining. Cells were cultured in a 6-well culture plate with cover glass coated with polylysine, then fixed with $4 \%$ paraformaldehyde for $30 \mathrm{~min}$, and washed with PBS 3 times. Hoechst 33342 staining $(5 \mu \mathrm{g} / \mathrm{ml})$ fluid was added for $10 \mathrm{~min}$ incubation at $37^{\circ} \mathrm{C}$, then wash with PBS 3 times. Observation and photography were performed under a fluorescence microscope.

Analysis of mitochondrial membrane potential. Mitochondrial membrane potential is one of the indicators to measure the mitochondrial function. The change of mitochondrial membrane potential was analyzed as previously described (15). The results were analyzed with Cell Quest ${ }^{\mathrm{TM}}$ analysis software (Becton-Dickinson, USA).

Analysis of cell cycle with flow cytometry. Trypsin was used for digestion and collection of the treated brain glioma cells, and the cells were fixed with $70 \%$ ethanol at $4^{\circ} \mathrm{C}$ overnight, then washed with PBS twice. PI synthetic staining solution (1 ml) (containing $10 \mu \mathrm{g}$ of RNase and $5 \mu \mathrm{l}$ of Triton X-100) was added for $30 \mathrm{~min}$ at $4^{\circ} \mathrm{C}$ in the dark before analysis with flow cytometry (Becton-Dickinson) for apoptosis.

Western blot assay. The brain glioma cells, of the experimental groups, were washed with PBS, and $2 \mathrm{ml}$ of protein lysis solution (Sigma) was added for cell lysis. The protein concentration was detemined. The protein was separated on a $10 \%$ SDS-PAGE gel. The proteins were transferred to PVDF membrane with a semidry method and sealed with 5\% skimmild powder. The first antibody was applied for $2 \mathrm{~h}$ before membrane bleaching with TBST. The second antibody was applied for $2 \mathrm{~h}$. Chemiluminesence was used for X-ray exposure imaging and strip scanning. Gray analysis was conducted using $\beta$-actin as the reference standard.

Statistical analysis. The experimental data were analyzed with SPSS 17.0 statistical software useing t-test and variance analysis. $\mathrm{p}<0.05$ was considered to indicate a significant statistical difference.

\section{Results}

Kankl gene expression is low in human brain glioma cells. RT-PCR and western blot assay were used to analyze the expressions of Kank1 gene and protein in U251 cells, U87 cells, HK-2 cells and GES-1 cells. It was found that the expressions of Kank1 gene and protein in U251 cells and U87 cells were both downregulated as compared with those of HK-2 cells and GES- 1 cells, and the downregulation was the most obvious in U87 cells. The above results meant that the expression of Kank1 gene and protein was low in U87 cells and U251 cells of human brain glioma cells (Fig. 1).

Silencing Kankl gene promotes human brain glioma cell proliferation. In this experiment, U87 cells and U251 cells were selected to analyze the impact of silencing the Kank1 gene on the cell proliferation capacity with MTT method so 
A

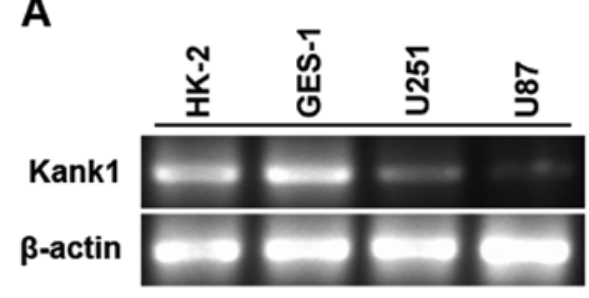

B

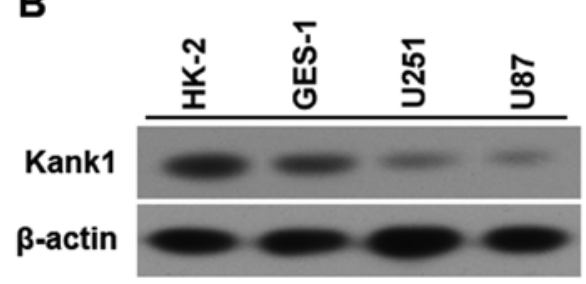

C

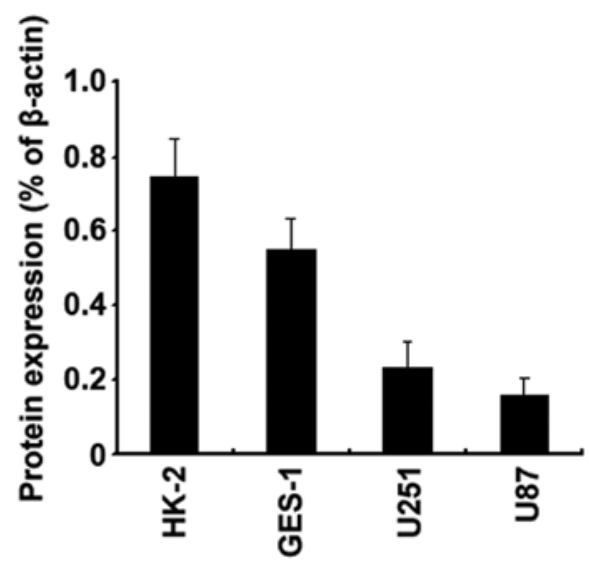

Figure 1. Expression of the Kank1 gene and protein in various cells. (A) RT-PCR analysis on Kank1 gene expressions in four cell types. (B) Western blot assay on Kank1 gene expressions in four cell types. (C) The results (B) were analyzed with Gel-Pro Analyzer 4.0 software. Each independent experimental sample was repeated 3 times.

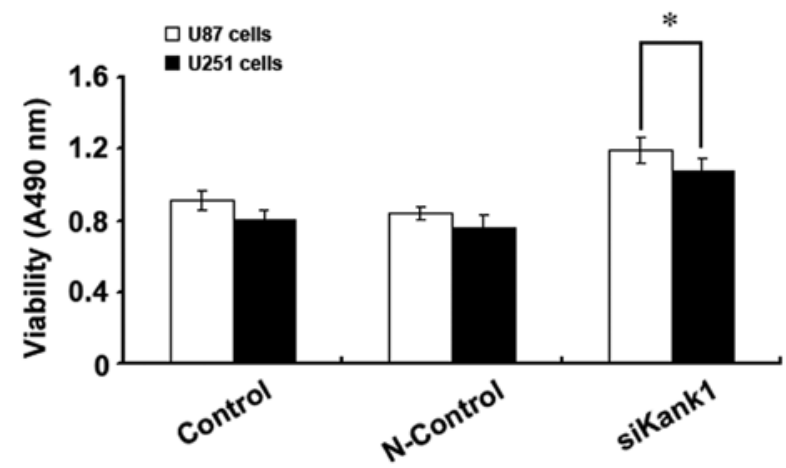

Figure 2. Silencing Kank1 gene to promote human brain glioma cell proliferation. MTT method for analysis to show significant increase of the proliferation capacity of the group of cells with Kank1 siRNA transfection as compared with untransfected group and the control group of transfection negative oligonucleotide. ${ }^{*} \mathrm{p}<0.05$. The data are from three independent experiments.

as to investigate the impact of the Kank1 gene on the growth of human brain glioma cells. It was found that the proliferation capacity of U87 and U251 cells increased drastically after transfection of the Kank1 siRNA for $48 \mathrm{~h}$ as compared with the control group and the control group of transfection negative oligonucleotides. This indicated that silencing Kank1 gene could significantly promote the proliferation capacity of human brain glioma cells (Fig. 2).

Upregulating Kankl gene inhibits the growth of human brain glioma cells. In order to further confirm the impact of Kank1 gene on the growth of human brain glioma cells, we successfully built and transfected Kank1 plasmid to upregulate the expression level of Kank1 gene in human brain glioma cells and used the MTT method for analysis. It was found that the proliferation capacity of U87 cells and U251 cells reduced drastically after upregulating Kank1 gene expression level as compared with the control group and the control group of transfection negative oligonucleotides. This indicated that

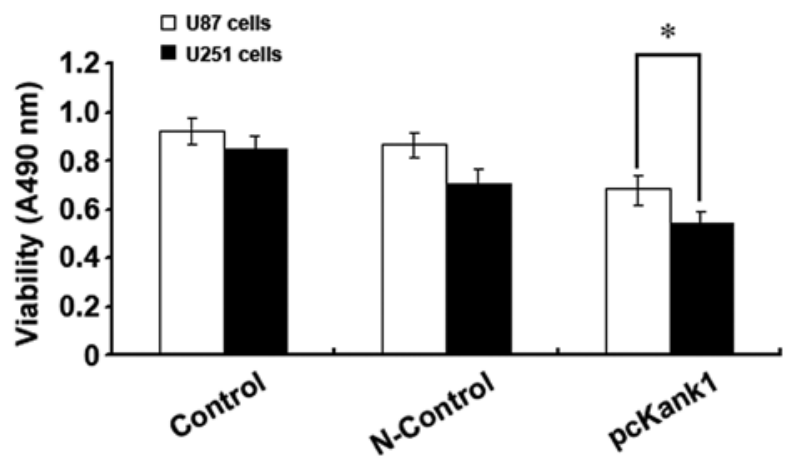

Figure 3. Impact of upregulation of the Kank1 gene on growth of human brain glioma cells. MTT method for analysis of the significant decline of the proliferation capacity of the group of cells with pcKank1 transfection as compared with untransfected group and the control group of transfection negative oligonucleotide. ${ }^{*} \mathrm{p}<0.05$. The data are from three independent experiments.

upregulating Kank1 gene expression could clearly inhibit the growth of human brain glioma cells (Fig. 3).

Upregulating Kankl gene induces the apoptosis of human brain glioma cells. In order to investigate whether Kank1 gene is relevant to the apoptosis of human brain glioma cells, we upregulated the Kank1 gene expression level and used Annexin V-FITC/PI double labeling method to analyze the apoptosis rate. It was found that the apoptosis rate of U87 cells and U251 cells increased drastically after upregulating Kank1 gene expression level as compared with the N-control group (Fig. 4A). The western blot assay indicated that the expression level of Procaspase- 9 and -3 proteins declined significantly (Fig. 4B and C). We used Hoechst 33342 staining for observation under the fluorescence microscope and found non-uniform agglutination of U87 cell chromatin with margination and pyknosis, or nuclear dense staining and formation of apoptosis bodies after upregulating Kank1 gene expression (Fig. 4D). The results indicated that upregulating 
A

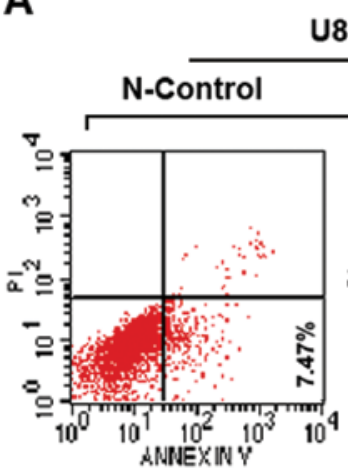

B

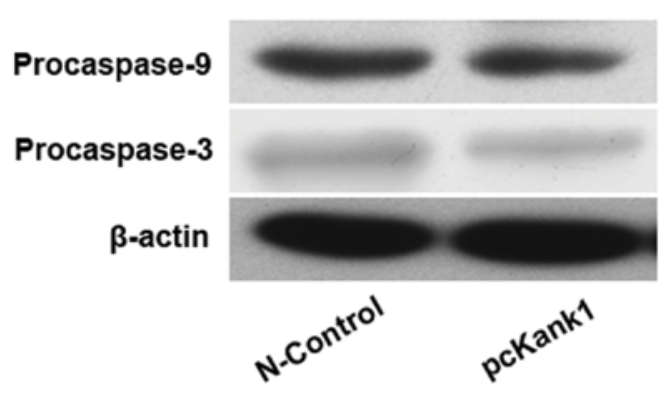

D

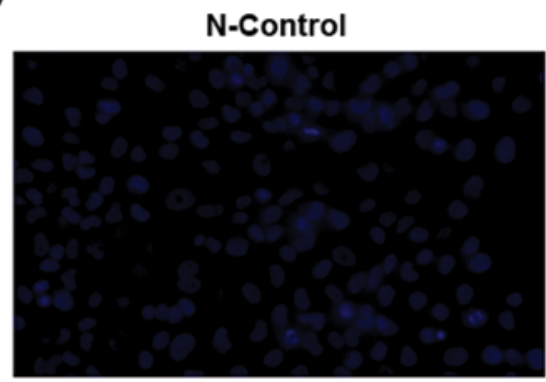

U87 cell

pcKank1
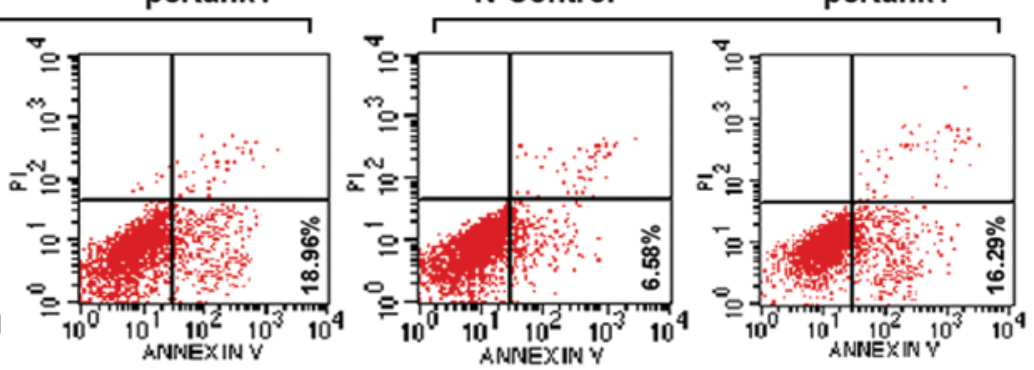

C
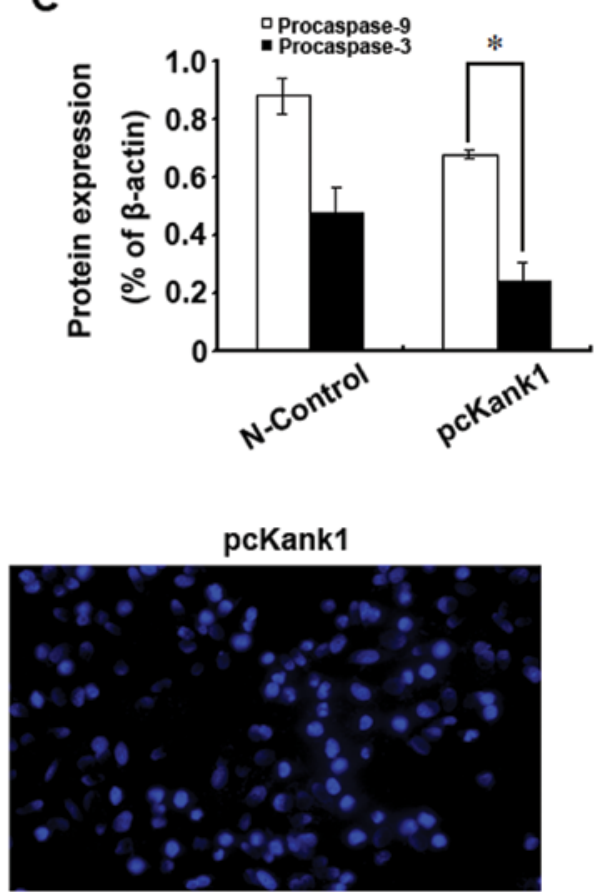

Figure 4. Upregulating the Kank1 gene results in apoptosis of human brain glioma cells. (A) Annexin V-FITC/PI double labeling to analyze apoptosis by flow cytometry. (B) Western blot assay to analyze expression of Procaspase- 9 and -3 proteins after upregulation of the Kank1 gene. (C) The western blot results were further analyzed using Gel-Pro Analyzer 4.0 software. (D) Hoechst 33342 staining to observe the apoptosis pattern (magnification x200). ${ }^{*}<<0.05$ as compared with the $\mathrm{N}$-control group. The data are from three independent experiments.

Kank1 gene may result in the apoptosis of human brain glioma cells.

Upregulation of the Kankl gene induces apoptosis of human brain glioma cells via mitochondria pathway. In order to investigate the relevant molecular mechanism of upregulating Kank1 gene-induced apoptosis of human brain glioma cells, we tested the change in the mitochondria membrane potential. It was found that upregulation of the Kank1 gene resulted in reduction of the mitochondria membrane potential (Fig. 5A). Additionally, we analyzed the mitochondria pathway apoptosis-relevant proteins $\mathrm{Bax}, \mathrm{Bcl}-2$ and cytochrome $\mathrm{C}$ via western blot assay. It was found that upregulating Kank1 gene resulted in an increase of Bax level of mitochondrion and a decline of Bcl-2 and cytochrome $\mathrm{C}$ level. However, the levels of intracytoplasm Bax, Bcl-2 and cytochrome $\mathrm{C}$ were the opposite to those of the mitochondrion (Fig. 5B-E). Our results indicated that upregulation of the Kank1 gene lowered the mitochondria membrane potential to promote the release of Bax and Bcl-2 as well as the release of cytochrome $\mathrm{C}$ into the cytoplasm so as to cause the apoptosis of human brain glioma cells.

Upregulation of the Kankl gene blocks the human brain glioma cells in G0/G1 phase. In order to further explore the mechanism of Kank1 gene-inhibited proliferation of human brain glioma cells, we analyzed the change in the cell cycle by flow cytometry. The ratio of human brain glioma cells in phase G0/G1 was much higher than that of the control group of transfection negative oligonucleotides after upregulation of the Kank1 gene (Fig. 6A). Also, the expression levels of cell cycle proteins CDK4, CDK6 and Cyclin D1 declined greatly after upregulation of the Kank1 gene (Fig. 6B and C). Our results show that upregulation of the Kank1 gene inhibited proliferation of human brain glioma cells possibly by blocking the cells in phase G0/ 
A

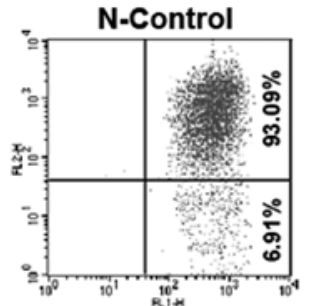

B

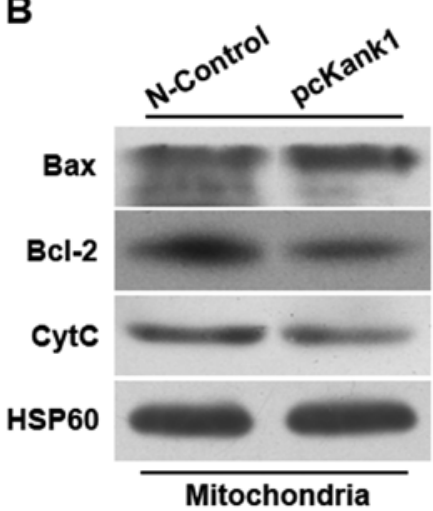

D

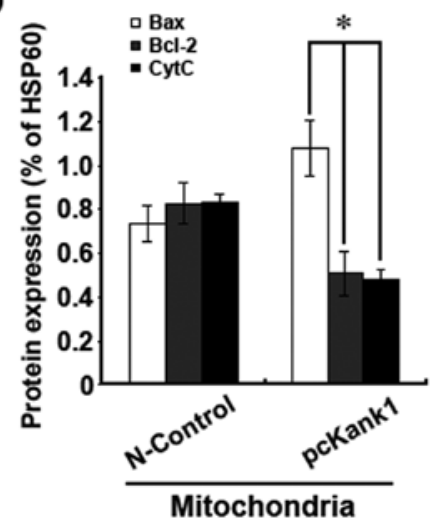

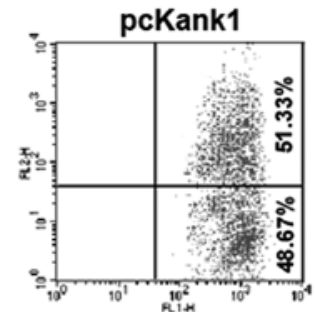

C

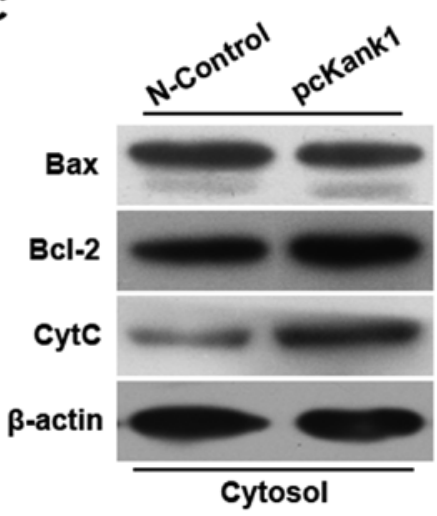

E

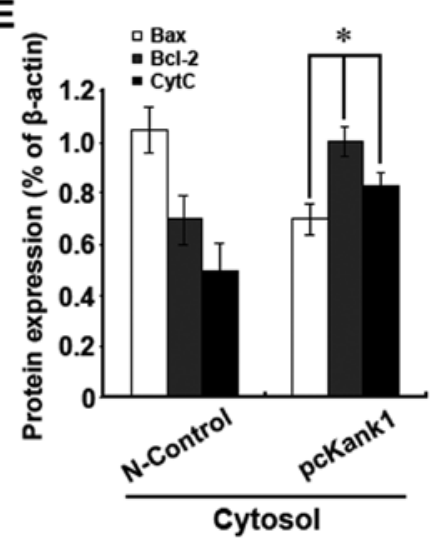

Figure 5. Upregulation of the Kank1 gene induces apoptosis of human brain glioma cells via mitochondria pathway. (A) JC-1 staining in flow cytometry analysis on the change in the mitochondria membrane potential. (B and C) Western blot assay to analyze expression of Bax, Bcl-2 and cytochrome $\mathrm{C}$ in the mitochondrion and the cytoplasm. (D and E) The results of (B and C) were analyzed with Gel-Pro Analyzer 4.0 software. "p<0.05 as compared with the $\mathrm{N}$-control group. Results are representative of three independent experiments.

G1 via downregulating the expression of the proteins CDK4, CDK6 and Cyclin D1.

\section{Discussion}

The Kank1 gene is an important member of the Kank gene family which is a candidate tumor suppressor gene verified in the renal cell carcinoma in 2002 (16). Located in human chromosome 9p24.3 with the total length of $27.7 \mathrm{kp}$, Kank1 gene contains 12 exons. Kank1 protein includes three parts, namely, conservative $\mathrm{N}$ terminal $\mathrm{KN}$ motif, intermediate coiled coil structural domain and $\mathrm{C}$ terminal ankyrin repeat domain. Ankyrin repeat domain and coiled coil structural domains are the functional domains for Kank1 protein to combine with other proteins to have a biological role (17). As a key adaptor protein, Kank1 is mainly distributed in the cytoplasm and forms a compound with $\beta$-catenin to shuttle in the nucleoplasm to adjust the $\beta$-catenin subcellular distribution. Kank1 plays an important role in the genesis, development, attack and metastasis of many malignant tumors $(7,18,19)$.

The objective of our study is to explore the expression of Kank1 gene in human brain glioma cells and its application value in treating human brain glioma. Our results show that the expression of Kank1 gene and protein were lower in human brain glioma cells, which means Kank1 gene is somehow closely related to the genesis and development of human brain glioma and may well be a potential therapeutic target for treating brain glioma. In order to further explore whether Kank1 gene is relevant to the genesis and development of human brain glioma and its detailed mechanism of action, we downregulated or upregulated the expression level of the Kank1 gene in human brain glioma cells and observed the biological changes of human brain glioma cells with deficiency or overexpression of the Kank1 gene. The proliferation of human brain glioma cells was found to increase signifi- 
A

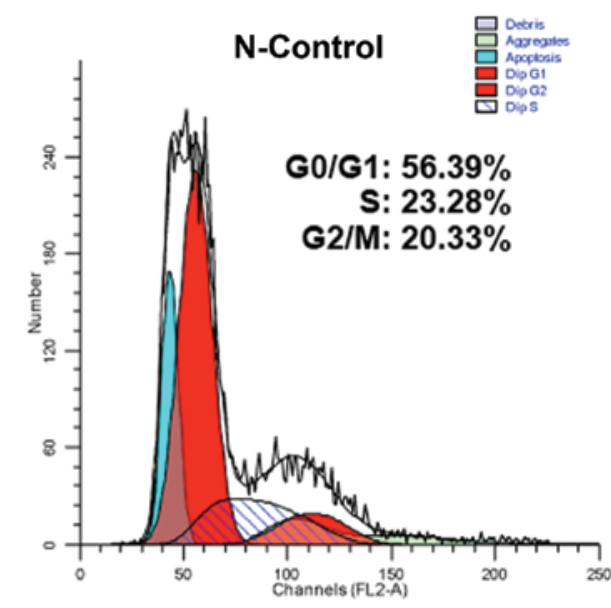

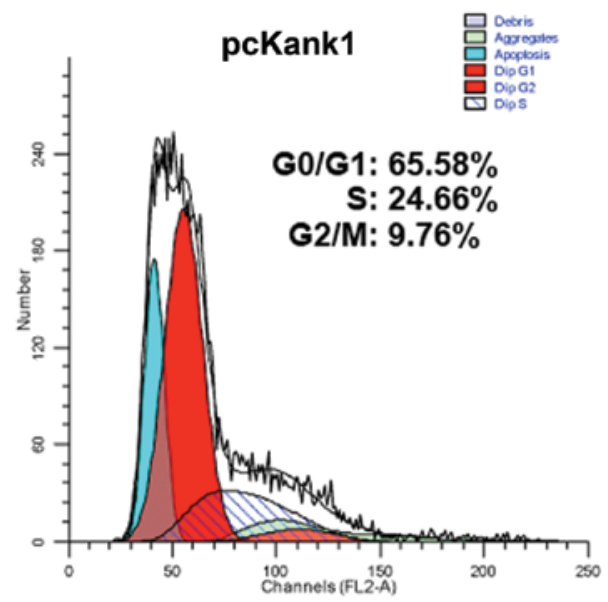

B

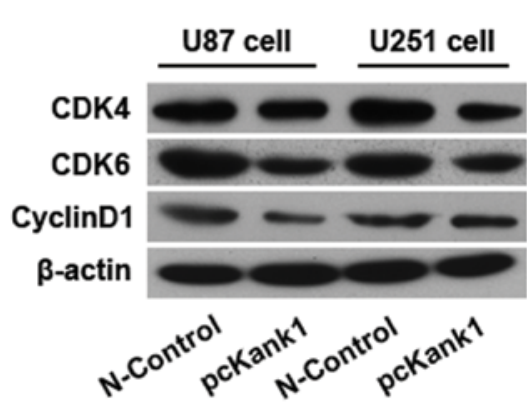

C

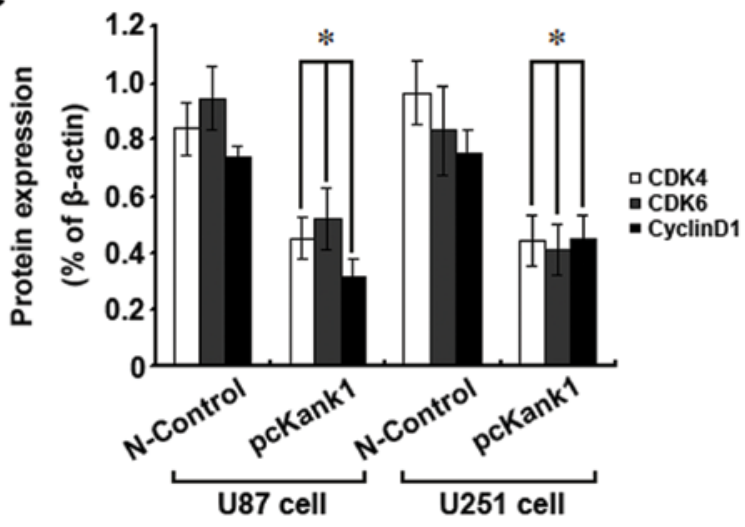

Figure 6. Upregulation of the Kank1 gene can block the human brain glioma cells in G0/G1 phase. (A) Analysis on the change in cell cycle by flow cytometry in U87 cells after upregulation of the Kank1 gene. (B) Western blot assay analysis on expression levels of proteins CDK4, CDK6 and Cyclin D1. (C) The western blot results were further analyzed using Gel-Pro Analyzer 4.0 software. "p $<0.05$, each independent experimental sample was repeated 3 times.

cantly after the Kank1 gene was downregulated. However, upregulating the Kank1 gene expression level could obviously inhibit the proliferation of human brain glioma cells and promote apoptosis. The cell cycle was blocked in G0/G1 phase. The above phenomena indicated that the low expression of Kank1 gene in human brain glioma cells promoted the proliferation of tumor cells. It was shown in previous studies that the low expression of Kank1 gene promotes the development of such malignant tumors as kidney cancer, cervical cancer, bladder cancer, prostate cancer, lung cancer and breast cancer (8-12). Our research findings agree with the results of the previous studies, which fully demonstrated that Kank1 gene also plays an important role of regulating in the proliferation of human brain glioma cells.

However, how Kank1 gene regulates the process of the proliferation of human brain glioma cells is still unknown. To explain this key issue, the investigation was carried out in terms of apoptosis and the cell cycle progress. The studies on Kank1 gene and apoptosis are still limited. Our findings show that upregulating Kank1 gene can result in the apoptosis of human brain glioma cells. Kakinuma et al (19) found in their research that Kank1 gene could control apoptosis via regulating the PI3K/AKT signal transduction pathway. Those results are similar to our findings. We found the inhibition of the proliferation of human brain glioma cells via upregulating Kank1 gene was closely related to apoptosis. Also, we found that upregulation of the Kank1 gene could block the cycle of human brain glioma cells in G0/G1 phase. The above phenomena indicated that the low expression of Kank1 gene in human brain glioma cells is necessary for the proliferation of tumor cells, and the upregulation of the Kank1 gene could inhibit the proliferation of human brain glioma cells.

It has already been proven in numerous studies that the mitochondria pathway and the death receptor pathway (20-22) are the classical paths for apoptosis of various tumor cells. Bcl-2 and Bax play a key role in the mitochondria apoptosis pathway (23-25). If Bax shifts from cytoplasm to mitochondria membrane, it can change the permeability of the mitochondrial membrane and promote cytochrome $\mathrm{C}$ to be released from the mitochondrion into the cytoplasm (26) to trigger the apoptosis cascade pathway finally resulting in apoptosis. We found in this study that upregulation of the Kank1 gene in human brain glioma cells resulted in the translocation of Bax and $\mathrm{Bcl}-2$ and the mitochondria 
membrane potential would change in turn for cytochrome $\mathrm{C}$ to be released from the mitochondrion into the cytoplasm finally causing apoptosis. It was confirmed by our research findings that the apoptosis of human brain glioma cells induced by upregulating Kank1 gene was closely related to the mitochondria pathway. Caspase family activation and cascade amplification role are the necessary prerequisite for apoptosis $(27,28)$. The expression levels of Procaspase-9 and -3 were found to reduce significantly after upregulation of the Kank1 gene in human brain glioma cells. It is speculated that the biological effect of activating Caspase-9 and -3 may be generated by the release of cytochrome $\mathrm{C}$ into the plasma in mitochondria apoptosis and it may play a key role in the apoptosis pathway, agreeing with the research findings of Riedl et al (29). Our above results confirmed that upregulation of the Kank1 gene may induce apoptosis of human brain glioma cells and it was closely related to the mitochondria pathway.

It is believed that the genesis of malignant tumors is closely related to the abnormal proliferation, apoptosis or reduction of the tumor cells. Therefore some research has proposed that it is possible to slow down or stop the growth of the tumor cells via promoting apoptosis of tumor cells in various ways $(30,31)$. It is undeniable that the cell apoptosis is often closely linked to the cell cycle. Previous studies show that the tumor cells may die or stop growing if the cells are blocked into a certain phase via various approaches $(32,33)$. Our results show that upregulating the Kank1 gene obviously inhibited the cycle progress of human brain glioma cells and blocked the cycle of the tumor cells into phase G0/G1. The expression levels of the cell cycle regulating proteins CDK4, CDK6 and Cyclin D1 also declined significantly. The above results indicated that upregulation of the Kank1 gene was able to change the cell cycle and inhibited the proliferation of human brain glioma cells in turn.

In conclusion, we found the lowering of Kank1 gene expression level in human brain glioma cells, and upregulation of the Kank1 gene was able to inhibit apoptosis of human brain glioma cells resulting in the blockade of the cell cycle via regulating $\mathrm{Bcl}-2 / \mathrm{Bax}$ to act on the mitochondria pathway. Kank1 gene may be used in the future to prove the theoretical basis for the clinical treatment of the human brain glioma.

\section{Acknowledgements}

This study was supported by the First Hospital of Jilin University.

\section{References}

1. Cordner R, Black KL and Wheeler CJ: Exploitation of adaptive evolution in glioma treatment. CNS Oncol 2: 171-179, 2013.

2. Hess KR, Broglio KR and Bondy ML: Adult glioma incidence trends in the United States, 1977-2000. Cancer 101: 2293-2299, 2004.

3. Eyüpoglu IY, Buchfelder M and Savaskan NE: Surgical resection of malignant gliomas - role in optimizing patient outcome. Nat Rev Neurol 9: 141-151, 2013.

4. Stummer W and Kamp MA: The importance of surgical resection in malignant glioma. Curr Opin Neurol 22: 645-649, 2009.

5. Mrugala MM: Advances and challenges in the treatment of glioblastoma: a clinician's perspective. Discov Med 15: 221-230, 2013.
6. Norden AD and Wen PY: Glioma therapy in adults. Neurologist 12: 279-292, 2006.

7. Clevers $\mathrm{H}$ : Wnt/beta-catenin signaling in development and disease. Cell 127: 469-480, 2006.

8. Demoulin JB, Medves S, Toffalini F, Essaghir A, Kallin A, Montano C, Velghe A and Duhoux F: Role of PDGF and FGF receptors in cancer. Bull Mem Acad R Med Belg 165: 310-315, 2010 (In French).

9. Kakinuma N, Zhu Y, Wang Y, Roy BC and Kiyama R: Kank proteins: structure, functions and diseases. Cell Mol Life Sci 66: 2651-2659, 2009

10. Lo KC, Stein LC, Panzarella JA, Cowell JK and Hawthorn L: Identification of genes involved in squamous cell carcinoma of the lung using synchronized data from DNA copy number and transcript expression profiling analysis. Lung Cancer 59: 315-331, 2008.

11. Sato M, Takahashi K, Nagayama K, Arai Y, Ito N, Okada M, Minna JD, Yokota J and Kohno T: Identification of chromosome arm $9 \mathrm{p}$ as the most frequent target of homozygous deletions in lung cancer. Genes Chromosomes Cancer 44: 405-414, 2005.

12. Kohno T, Otsuka A, Girard L, Sato M, Iwakawa R, Ogiwara H, Sanchez-Cespedes M, Minna JD and Yokota J: A catalog of genes homozygously deleted in human lung cancer and the candidacy of PTPRD as a tumor suppressor gene. Genes Chromosomes Cancer 49: 342-352, 2010.

13. Chan WH: Citrinin induces apoptosis in mouse embryonic stem cells. IUBMB Life 60: 171-179, 2008.

14. Tomiyama A, Tachibana K, Suzuki K, Seino S, Sunayama J, Matsuda KI, Sato A, Matsumoto Y, Nomiya T, Nemoto K, Yamashita H, Kayama T, Ando K and Kitanaka C: MEK-ERKdependent multiple caspase activation by mitochondrial proapoptotic Bcl-2 family proteins is essential for heavy ion irradiation-induced glioma cell death. Cell Death Dis 1: e60, 2010.

15. Tang B, Zhang Y, Liang R, Yuan P, Du J, Wang H and Wang L: Activation of the $\delta$-opioid receptor inhibits serum deprivationinduced apoptosis of human liver cells via the activation of PKC and the mitochondrial pathway. Int J Mol Med 28: 1077-1085, 2011.

16. Sarkar S, Roy BC, Hatano N, Aoyagi T, Gohji K and Kiyama R: A novel ankyrin repeat-containing gene (Kank) located at 9p24 is a growth suppressor of renal cell carcinoma. J Biol Chem 277: 36585-36591, 2002.

17. Zhu Y, Kakinuma N, Wang Y and Kiyama R: Kank proteins: a new family of ankyrin-repeat domain-containing proteins. Biochim Biophys Acta 1780: 128-133, 2008.

18. Roy BC, Kakinuma N and Kiyama R: Kank attenuates actin remodeling by preventing interaction between IRSp53 and Rac1. J Cell Biol 184: 253-267, 2009.

19. Kakinuma N, Roy BC, Zhu Y, Wang Y and Kiyama R: Kank regulates RhoA-dependent formation of actin stress fibers and cell migration via 14-3-3 in PI3K-Akt signaling. J Cell Biol 181: 537-549, 2008

20. Matthews GM, Newbold A and Johnstone RW: Intrinsic and extrinsic apoptotic pathway signaling as determinants of histone deacetylase inhibitor antitumor activity. Adv Cancer Res 116: 165-197, 2012.

21. Nieminen AI, Partanen JI and Klefstrom J: c-Myc blazing a trail of death: coupling of the mitochondrial and death receptor apoptosis pathways by c-Myc. Cell Cycle 6: 2464-2472, 2007.

22. Ghobrial IM, Witzig TE and Adjei AA: Targeting apoptosis pathways in cancer therapy. CA Cancer J Clin 55: 178-194, 2005.

23. Renault TT, Teijido O, Antonsson B, Dejean LM and Manon S: Regulation of Bax mitochondrial localization by Bcl-2 and $\mathrm{Bcl}-\mathrm{x}(\mathrm{L})$ : keep your friends close but your enemies closer. Int J Biochem Cell Biol 45: 64-67, 2013.

24. Mattson MP and Kroemer G: Mitochondria in cell death: novel targets for neuroprotection and cardioprotection. Trends Mol Med 9: 196-205, 2003

25. Lindsay J, Esposti MD and Gilmore AP: Bcl-2 proteins and mitochondria - specificity in membrane targeting for death. Biochim Biophys Acta 1813: 532-539, 2011.

26. Saito M, Korsmeyer SJ and Schlesinger PH: BAX-dependent transport of cytochrome $c$ reconstituted in pure liposomes. Nat Cell Biol 2: 553-555, 2000.

27. Yuan S and Akey CW: Apoptosome structure, assembly, and procaspase activation. Structure 21: 501-515, 2013. 
28. McIlwain DR, Berger T and Mak TW: Caspase functions in cell death and disease. Cold Spring Harb Perspect Biol 5: a008656, 2013.

29. Riedl SJ and Shi Y: Molecular mechanisms of caspase regulation during apoptosis. Nat Rev Mol Cell Biol 5: 897-907, 2004.

30. Williams GT: Programmed cell death: apoptosis and oncogenesis. Cell 65: 1097-1098, 1991.

31. Evan G and Littlewood T: A matter of life and cell death. Science 281: 1317-1322, 1998.
32. Wang XM, Cui JW, Li W, Cai L, Song W and Wang GJ: Silencing of the COPS3 gene by siRNA reduces proliferation of lung cancer cells most likely via induction of cell cycle arrest and apoptosis. Asian Pac J Cancer Prev 13: 1043-1048, 2012.

33. Powathil GG, Gordon KE, Hill LA and Chaplain MA: Modelling the effects of cell-cycle heterogeneity on the response of a solid tumour to chemotherapy: biological insights from a hybrid multiscale cellular automaton model. J Theor Biol 308: 1-19, 2012. 